\title{
Complex Codon Usage Pattern and Compositional Features of Retroviruses
}

\author{
Sourav RoyChoudhury ${ }^{1,2}$ and Debaprasad Mukherjee ${ }^{1,3}$ \\ ${ }^{1}$ School of Information Technology, Bengal Engineering and Science University, Shibpur, Howrah, West Bengal 711103, India \\ ${ }^{2}$ School of Medical Science and Technology, Indian Institute of Technology Kharagpur, Kharagpur-721302, India \\ ${ }^{3}$ Department of Information Technology, Dr. B.C. Roy Engineering College, West Bengal University of Technology, \\ Durgapur, West Bengal 713206, India
}

Correspondence should be addressed to Debaprasad Mukherjee; mdebaprasad@gmail.com

Received 30 May 2013; Revised 5 September 2013; Accepted 7 September 2013

Academic Editor: Kazuhisa Nishizawa

Copyright (C) 2013 S. RoyChoudhury and D. Mukherjee. This is an open access article distributed under the Creative Commons Attribution License, which permits unrestricted use, distribution, and reproduction in any medium, provided the original work is properly cited.

\begin{abstract}
Retroviruses infect a wide range of organisms including humans. Among them, HIV-1, which causes AIDS, has now become a major threat for world health. Some of these viruses are also potential gene transfer vectors. In this study, the patterns of synonymous codon usage in retroviruses have been studied through multivariate statistical methods on ORFs sequences from the available 56 retroviruses. The principal determinant for evolution of the codon usage pattern in retroviruses seemed to be the compositional constraints, while selection for translation of the viral genes plays a secondary role. This was further supported by multivariate analysis on relative synonymous codon usage. Thus, it seems that mutational bias might have dominated role over translational selection in shaping the codon usage of retroviruses. Codon adaptation index was used to identify translationally optimal codons among genes from retroviruses. The comparative analysis of the preferred and optimal codons among different retroviral groups revealed that four codons GAA, AAA, AGA, and GGA were significantly more frequent in most of the retroviral genes inspite of some differences. Cluster analysis also revealed that phylogenetically related groups of retroviruses have probably evolved their codon usage in a concerted manner under the influence of their nucleotide composition.
\end{abstract}

\section{Introduction}

The retroviruses are a diverse family of enveloped single stranded retro transcribing RNA viruses unique for their use of reverse transcription of the viral RNA into linear double stranded DNA during replication and the subsequent integration of the DNA into the host genome. Members of this family cause diseases in a wide range of organisms, including humans [1]. Human immunodeficiency virus 1 (HIV1) is responsible for acquired immunodeficiency syndrome (AIDS) and is largely dependent on transmission of contaminated body fluids during sexual intercourse, pregnancy, and so forth [2]. More than 30 million people worldwide are living with HIV. Besides, retroviruses are increasingly becoming valuable tools in molecular biology and have been used successfully in gene therapy [3]. Based on morphology, pathogenicity, and molecular phylogenetics, retroviruses have been classified into two subfamilies: Orthoretrovirinae, Spumaretrovirinae and rest of the viruses are unclassified. The Orthoretrovirinae is further classified into six genera: Alpharetrovirus, Betaretrovirus, Deltaretrovirus, Epsilonretrovirus, Gammaretrovirus, and Lentivirus.

The analysis of codon usage of whole organisms and/or organisms from closely related groups of them reveals trends and anomalies in the choice and bias in the frequency of codons and related nucleotide composition, including evolutionary features. Synonymous codons do not occur in equal frequency in genes and genomes. The relative frequency of these synonymous codons in the genes varies significantly in a nonrandom manner between species, even between those from the same taxon due to a complex balance between mutational bias, various selection forces (e.g., translational selection), and drift acting on the genes or genomes [4]. Codon and base usage patterns reveal information on the nature 
of molecular evolution of genes and genomes, sometimes even events of horizontal gene transfer. Evidence exists of correlations between codon usage bias and nucleotide composition in some viruses, clearly indicating that mutational bias towards particular nucleotide content influences general codon usage of organisms [5]. For example, in free living organisms, such as E. coli, S. cerevisiae, C. elegans, D. melanogaster, and $A$. thaliana, knowledge of codon usage bias gives insights into the content of the isoacceptor tRNAs in genomes, their coadaptation, and potential levels of gene expression due to selection for translational efficiency $[6,7]$.

In this study, the codon usage patterns of all the available 56 sequenced retroviruses' genomes (from GenBank) containing 246 ORFs (longer than $150 \mathrm{bp}$ ) were analyzed. Results from this study would be useful for revealing retroviral gene composition and evolution and additionally may be useful in selecting appropriate host expression systems to improve the expression of target genes in vivo and in vitro for the design of gene delivery and expression systems for use in gene therapy and immunization.

\section{Materials and Methods}

56 completely sequenced retroviral genomes were available from NCBI GenBank (February 2010). These belonged to two major subfamilies: Orthoretrovirinae, Spumaretrovirinae and rest of the viruses were unclassified. Six viruses belong to Spumaretrovirinae, while 3 viruses were unclassified. The remaining 47 viruses belong to Orthoretrovirinae subfamily. Six genera are present within Orthoretrovirinae, namely: Alpharetrovirus, Betaretrovirus, Deltaretrovirus, Epsilonretrovirus, Gammaretrovirus, and Lentivirus. Among the 47 Orthoretrovirinae viruses, there are 7 Alpharetroviruses, 6 Betaretroviruses, 8 Deltaretroviruses, 2 Epsilonretroviruses, 14 Gammaretroviruses, and 10 Lentiviruses. 246 ORFs corresponding to all the completely sequenced genomes were available in GenBank. Only the genes with a length greater than or equal to $150 \mathrm{bp}$ have been considered for further study. All these sequences together contained 135,304 of codons.

The various statistical parameters characterizing synonymous codon frequency, codon bias, base composition of whole genes, base composition at 3rd codon positions, relative gene expression levels, preferred and optimal codons, correspondence and cluster analysis on codon usage, and the associated means, standard deviations (SD), correlation coefficients, and chi-square statistics $\left(\chi^{2}\right)$ were computed using CodonW [8], GCUA [9], and STATISTICA 8.0 (http://www .statsoft.com/).

\section{Results}

3.1. Codon and Nucleotide Bias. The "Effective Number of Codons" (ENc) of a gene sequence measures the degree of bias in codon usage in the gene [10]. It ranges from 20 to 61, with values below 35 implying high bias while above 50 implying low bias. It is found that none of the retroviral genes had any strong codon bias. Around 50\% of the genes had weak codon bias, implying that the rest half of the genes are moderately/randomly biased. Approximately $80 \%$ of the moderately biased genes belong to Orthoretorvirinae, 50\% belong to Lentivirus, and $13 \%$ to Deltaretrovirus. Spumaretorvirinae and "other Orthoretrovirinae groups" contain some amount of the moderately biased genes (Table 1). Some retroviruses show more codon bias than the others. These are FIV, SFV-3, VISNA, OLV, and HIV-1 whose average ENc lies between 40 and 45 . All of these are Lentiviruses, except SFV-3 which belongs to Spumaretorvirinae. The total range of ENc for all the retroviruses taken together was from 40 to 60 .

Nucleotide preferences are usually an indication for the nature of mutational bias in genes or genomes. Here, in retroviruses, explicit differences are observed in nucleotide preferences. The AU content (overall $\mathrm{A}+\mathrm{U}$ ) of genes in single retrovirus ranged from $35 \%$ to about $60 \%$ (Table 1). AU3 (A + $\mathrm{U}$ content of the 3 rd synonymous codon position) content in retroviruses varied over a large range, from about $20 \%$ to $75 \%$. FIV had the highest, both AU (60\%) and AU3 (70\%) content. FFV, SFV-3, VISNA, OLV, and HIV-1 also had similar levels of high AU content. These viruses also had relatively higher codon bias among the whole set of retroviruses. ACMHV-2 had the lowest AU (35\%) and AU3 (20\%) content. Among all the retroviruses, FuSV, Y73SV, AMCV, WMSV, HTLV4, HTLV-1, and STLV-2 had low AU (35 to 45\%) and AU3 (20 to $45 \%$ ) content. It was observed that AU content of the genes correlated strongly with their AU3 content $(r=0.91$, $P<0.0001$ ). AU1 and AU2 content (AU contents at first and second codon positions of genes, resp.) of retroviral genes are both about $50 \%$. This indicates an almost equal preference for $\mathrm{AU}$ and GC in both the first and second codon positions. But, in general, AU12 content (AU1 + AU2) is notably less than AU3 of retroviral genes as a whole.

When ENc versus AU3 content is plotted for the whole dataset, it is seen that only a small number of genes lie on the expected curve (the curve representing the variation of codon bias when determined by base composition only), while majority of the genes with low ENc values were lying well below it (Figure 1(a)). In the viruses with relatively higher codon bias, most of the genes with moderate codon bias have high AU3 (i.e., low GC) content. Furthermore, while considering the length of retroviral genes, it was found that no significant correlation existed between it and ENc of genes.

3.2. Preferred and Optimal Codons. Codons occurring in high frequencies in the total codon usage data of an organism are called preferred codons. Here, in retroviruses, significant differences (using $\chi^{2}$ test) in overall codon usage frequencies were observed between the pairwise combinations of retroviral clades. But some pairs of viruses-Betaretrovirus and Spumaretrovirinae/Epsilonretrovirus; Epsilonretrovirus and Betaretrovirus/Lentivirus or other unclassified retroviruses; and Gammaretrovirus and Deltaretrovirus or the remaining unclassified retroviruses-were exceptions and did not show significant differences in their overall codon usage frequencies $(P>0.05)$. Four codons, GAA (Glu), AAA (Lys), AGA (Arg), and GGA (Gly), were particularly preferred to a large extent in retroviruses. Seven other codons, UUU (Phe), UUA (Leu), UAU (Tyr), CAA (Gln), AAU (Asn), 
TABLE 1: Characteristics and codon usage pattern and AU distribution of retroviral genomes (shown in clades).

\begin{tabular}{|c|c|c|c|c|c|c|c|c|}
\hline Virus* & Abbrev. names & Accn. number & Mean ENc & $\mathrm{SD}$ & Mean AU \% & $\mathrm{SD}$ & Mean AU3 \% & SD \\
\hline \multicolumn{9}{|l|}{ Orthoretrovirinae } \\
\hline \multicolumn{9}{|l|}{ Alpharetrovirus } \\
\hline Avian carcinoma virus & ACMHV-2 & NC_001402 & 40.51 & 0.00 & 36.20 & 0.00 & 18.50 & 0.00 \\
\hline Avian leukosis virus-RSA & ALV-A & NC_001408 & 57.09 & 3.32 & 44.80 & 7.35 & 45.23 & 9.32 \\
\hline Avian myelocytomatosis virus & AMCV & NC_001866 & 52.79 & 0.00 & 39.60 & 0.00 & 32.00 & 0.00 \\
\hline Fujinami sarcoma virus & FuSV & NC_001403 & 42.61 & 0.00 & 37.90 & 0.00 & 22.20 & 0.00 \\
\hline Rous sarcoma virus & RSV & NC_001407 & 53.50 & 9.93 & 44.90 & 5.28 & 41.90 & 15.25 \\
\hline UR2 sarcoma virus & UR2SV & NC_001618 & 55.68 & 0.59 & 53.40 & 0.00 & 56.65 & 1.77 \\
\hline Y73 sarcoma virus & Y73SV & NC_008094 & 39.41 & 0.00 & 38.20 & 0.00 & 21.20 & 0.00 \\
\hline \multicolumn{9}{|l|}{ Betaretrovirus } \\
\hline Enzootic nasal tumour virus of goats & ENTV-2 & NC_004994 & 49.86 & 1.36 & 57.63 & 2.33 & 70.20 & 1.53 \\
\hline Jaagsiekte sheep retrovirus & JSRV & NC_001494 & 49.25 & 4.26 & 58.58 & 2.38 & 66.08 & 9.57 \\
\hline Mason-Pfizer monkey virus & MPMV & NC_001550 & 50.10 & 1.31 & 57.58 & 1.27 & 68.13 & 1.60 \\
\hline Mouse mammary tumor virus & MMTV & NC_001503 & 51.84 & 2.16 & 55.98 & 0.62 & 62.80 & 2.42 \\
\hline Ovine enzootic nasal tumour virus & ENTV-1 & NC_007015 & 49.23 & 2.80 & 58.28 & 2.72 & 71.45 & 2.87 \\
\hline Squirrel monkey retrovirus $-H L B$ & SMRV-HLB & NC_001514 & 53.01 & 4.61 & 51.68 & 2.01 & 54.78 & 3.39 \\
\hline \multicolumn{9}{|l|}{ Deltaretrovirus } \\
\hline Bovine leukemia virus & BLV & NC_001414 & 52.05 & 2.54 & 44.98 & 3.49 & 47.35 & 4.83 \\
\hline \multicolumn{9}{|l|}{ Primate T-lymphotropic virus 1} \\
\hline Human T-lymphotropic virus 1 & HTLV-1 & NC_001436 & 50.88 & 1.62 & 46.27 & 2.05 & 45.35 & 2.82 \\
\hline Simian T-lymphotropic virus 1 & STLV-1 & NC_000858 & 51.09 & 2.06 & 46.73 & 2.29 & 45.80 & 3.45 \\
\hline \multicolumn{9}{|l|}{ Primate T-lymphotropic virus 2} \\
\hline Human T-lymphotropic virus 2 & HTLV-2 & NC_001488 & 50.04 & 1.86 & 45.58 & 3.31 & 44.46 & 3.11 \\
\hline Simian T-lymphotropic virus 2 & STLV-2 & NC_001815 & 51.19 & 4.24 & 43.08 & 3.82 & 41.97 & 5.16 \\
\hline Human T-lymphotropic virus 4 & HTLV-4 & NC_011800 & 50.86 & 2.71 & 42.85 & 2.62 & 40.68 & 2.23 \\
\hline Simian T-cell lymphotropic virus 6 & STLV-6 & NC_011546 & 54.01 & 3.83 & 47.00 & 2.78 & 48.73 & 4.68 \\
\hline Simian T-lymphotropic virus 3 & STLV-3 & NC_003323 & 55.39 & 3.72 & 44.92 & 2.73 & 44.52 & 2.31 \\
\hline \multicolumn{9}{|l|}{ Epsilonretrovirus } \\
\hline Snakehead retrovirus & SnRV & NC_001724 & 51.88 & 7.91 & 50.31 & 4.73 & 58.46 & 4.80 \\
\hline Walleye dermal sarcoma virus & WDSV & NC_001867 & 53.51 & 2.45 & 57.82 & 3.39 & 65.50 & 3.56 \\
\hline \multicolumn{9}{|l|}{ Gammaretrovirus } \\
\hline Abelson murine leukemia virus & AbMLV & NC_001499 & 55.03 & 6.45 & 46.73 & 8.81 & 46.50 & 11.23 \\
\hline Feline leukemia virus & FeLV & NC_001940 & 53.84 & 4.16 & 50.30 & 1.70 & 53.60 & 0.99 \\
\hline \multicolumn{9}{|l|}{ Murine leukemia virus } \\
\hline Friend murine leukemia virus & FrMLV & NC_001362 & 54.95 & 1.03 & 46.70 & 1.41 & 47.70 & 1.82 \\
\hline Moloney murine leukemia virus & MoMLV & NC_001501 & 54.72 & 0.10 & 47.00 & 1.84 & 48.77 & 2.80 \\
\hline Murine type $C$ retrovirus & MTCR & NC_001702 & 52.33 & 3.00 & 45.90 & 0.99 & 45.50 & 2.44 \\
\hline Rauscher murine leukemia virus & R-MuLV & NC_001819 & 55.42 & 0.62 & 46.87 & 1.40 & 47.60 & 1.32 \\
\hline Gibbon ape leukemia virus & GALV & NC_001885 & 55.83 & 1.34 & 47.57 & 1.16 & 48.83 & 0.67 \\
\hline Moloney murine sarcoma virus & MOMSV & NC_001502 & 56.40 & 3.24 & 47.60 & 6.26 & 46.08 & 8.42 \\
\hline Murine osteosarcoma virus & MuSV & NC_001506 & 49.97 & 0.98 & 42.65 & 1.77 & 37.35 & 7.14 \\
\hline RD114 retrovirus & RD-114 & NC_009889 & 54.31 & 1.93 & 49.70 & 3.68 & 51.60 & 3.25 \\
\hline Reticuloendotheliosis virus & REV & NC_006934 & 57.09 & 0.20 & 46.90 & 0.80 & 46.50 & 1.44 \\
\hline Spleen focus-forming virus & SFFV & NC_001500 & 51.03 & 5.85 & 45.27 & 2.28 & 47.33 & 3.37 \\
\hline Woolly monkey sarcoma virus & WMSV & NC_009424 & 51.39 & 8.65 & 42.60 & 4.04 & 37.97 & 13.23 \\
\hline Xenotropic MuLV-related virus VP62 & XMRV-VP62 & NC_007815 & 52.79 & 2.41 & 46.43 & 1.29 & 46.80 & 2.18 \\
\hline \multicolumn{9}{|l|}{ Lentivirus } \\
\hline Bovine immunodeficiency virus & BIV & NC_001413 & 53.22 & 4.43 & 53.26 & 3.60 & 57.90 & 5.35 \\
\hline Caprine arthritis-encephalitis virus & CAEV & NC_001463 & 45.93 & 6.96 & 57.10 & 3.45 & 67.63 & 4.46 \\
\hline Equine infectious anemia virus & EIAV & NC_001450 & 47.05 & 7.93 & 59.25 & 4.56 & 67.43 & 1.18 \\
\hline Feline immunodeficiency virus & FIV & NC_001482 & 43.87 & 6.58 & 62.01 & 3.83 & 71.39 & 9.91 \\
\hline Human immunodeficiency virus 1 & HIV-1 & NC_001802 & 45.05 & 4.01 & 55.49 & 4.66 & 64.78 & 7.24 \\
\hline
\end{tabular}


TABle 1: Continued.

\begin{tabular}{|c|c|c|c|c|c|c|c|c|}
\hline Virus $^{*}$ & Abbrev. names & Accn. number & Mean ENc & $\mathrm{SD}$ & Mean AU \% & $\mathrm{SD}$ & Mean AU3 \% & SD \\
\hline Human immunodeficiency virus 2 & HIV-2 & NC_001722 & 52.43 & 5.73 & 51.49 & 3.02 & 56.51 & 4.77 \\
\hline Ovine lentivirus & OLV & NC_001511 & 44.59 & 4.47 & 57.72 & 3.78 & 65.73 & 6.90 \\
\hline \multicolumn{9}{|l|}{ Simian immunodeficiency virus } \\
\hline Simian immunodeficiency virus & SIV & NC_001549 & 48.80 & 4.12 & 54.73 & 3.32 & 60.62 & 7.82 \\
\hline Simian immunodeficiency virus SIV-mnd 2 & SIV-mnd-2 & NC_004455 & 51.44 & 5.83 & 54.91 & 2.63 & 58.94 & 6.43 \\
\hline Visna/Maedi virus & VISNA & NC_001452 & 44.47 & 5.34 & 57.83 & 2.70 & 68.25 & 6.51 \\
\hline \multicolumn{9}{|l|}{ Spumaretrovirinae } \\
\hline Bovine foamy virus & $\mathrm{BFV}$ & NC_001831 & 55.93 & 2.36 & 52.14 & 3.66 & 58.76 & 5.22 \\
\hline Equine foamy virus & EFV & NC_002201 & 45.53 & 2.69 & 58.00 & 4.83 & 71.16 & 5.47 \\
\hline Feline foamy virus & FFV & NC_001871 & 48.58 & 3.78 & 60.53 & 2.95 & 70.40 & 3.59 \\
\hline Macaque simian foamy virus & SFVmac & NC_010819 & 46.02 & 3.01 & 58.52 & 4.92 & 73.60 & 5.31 \\
\hline Simian foamy virus & SFV & NC_001364 & 47.94 & 3.91 & 58.03 & 4.38 & 70.58 & 8.48 \\
\hline Simian foamy virus 3 & SFV-3 & NC_010820 & 44.33 & 3.23 & 59.50 & 5.31 & 75.20 & 4.35 \\
\hline \multicolumn{9}{|l|}{ Unclassified retroviruses } \\
\hline Atlantic salmon swim bladder sarcoma virus & SSSV & NC_007654 & 54.69 & 2.28 & 51.70 & 3.54 & 57.90 & 6.65 \\
\hline Avian endogenous retrovirus EAV-HP & EAV-HP & NC_005947 & 59.38 & 0.00 & 47.60 & 0.00 & 47.80 & 0.00 \\
\hline Xenopus laevis endogenous retrovirus Xen1 & Xen-1 & NC_010955 & 59.60 & 1.80 & 53.35 & 2.76 & 58.00 & 4.11 \\
\hline
\end{tabular}

${ }^{*}$ Viruses are shown in their respective genera.

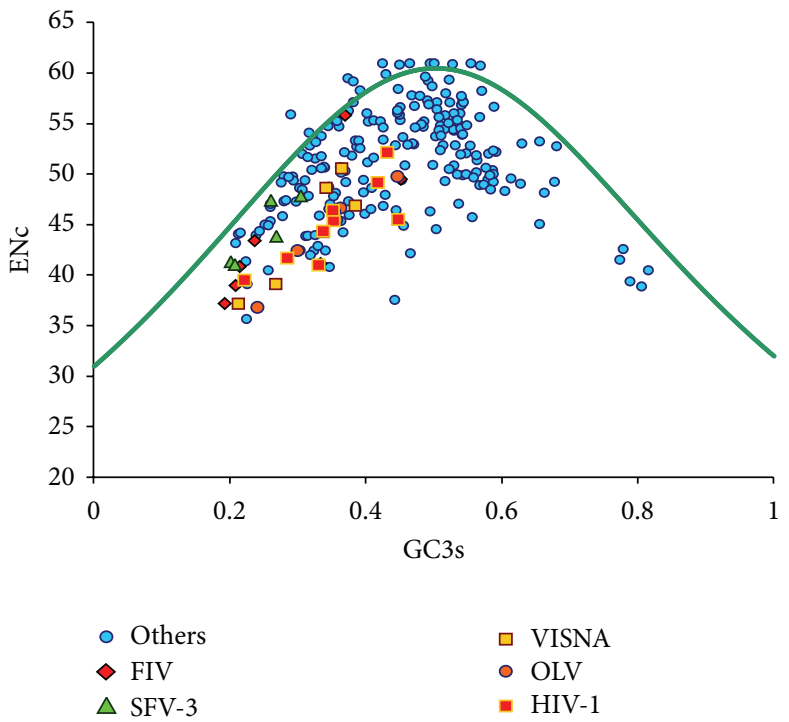

(a)

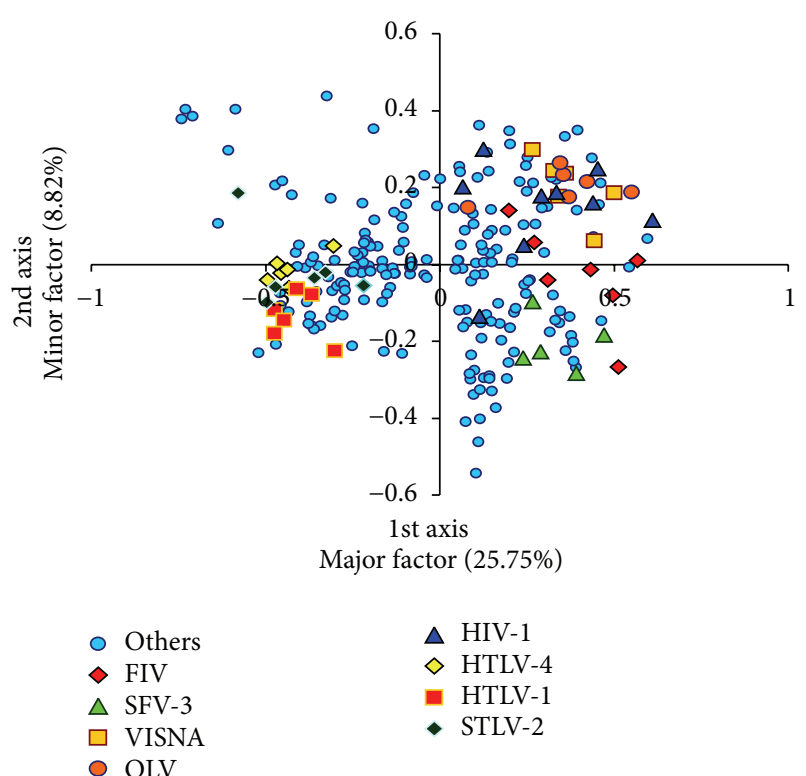

(b)

Figure 1: (a) ENc versus GC3 plot of all the genes. The reference viruses are in different colors. (b) The values of the first axis and the second axis of each gene in CoA. Genes from reference retroviruses are shown in different colors; genes from other viruses are plotted in blue colour.

GAU (Asp), and UGU (Cys), are also frequently preferred (see Supplementary Material available online at http://dx.doi.org/10.1155/2013/848123). It was also observed that closely related viruses, for example, those within the Orthoretrovirinae subfamily and those which are phylogenetically relatively closer to this group, prefer similar set of codons. It was observed especially among the viruses within genera like Betaretrovirus, Deltaretrovirus, Spumaretrovirinae, and Lentivirus. On the other hand, the Alpharetroviruses and Gammaretroviruses were somewhat of an exception with less similarity in their set of preferred codons. In general, the preferred codons almost always had $\mathrm{A}$ or $\mathrm{U}$ at their 3rd synonymous codon positions (in Betaretrovirus, Epsilonretrovirus, Lentivirus, and Spumaretrovirinae). But some viruses (Alpharetrovirus, Gammaretrovirus, and Deltaretrovirus) were exceptions, with high $\mathrm{G}$ or $\mathrm{C}$ content at their 3rd codon positions of preferred codons. It is also observed that the choice of preferred codons correlated with the overall 


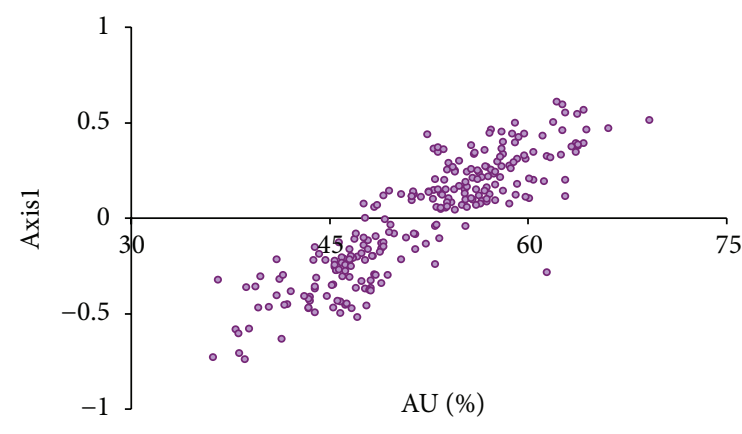

(a)

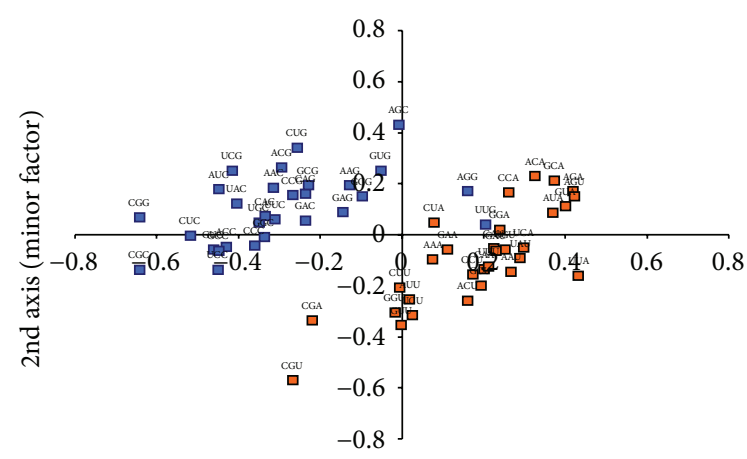

1 st axis (major factor)

- 3rd codon position $\mathrm{C} / \mathrm{G}$

- 3rd codon position $\mathrm{A} / \mathrm{U}$

(b)

FIgURE 2: (a) Correlation between AU content of each retroviral gene and their position on the first axis of CoA. (b) The distribution of synonymous codons is shown along the first and second axes of the CoA. Codons ending with $\mathrm{G}$ or $\mathrm{C}$ are shown in blue colors, and codons ending with $\mathrm{A}$ or $\mathrm{U}$ are shown in orange colour.

genomic composition of the viruses. AU rich genomes preferred AU ending codons, and GC rich genomes preferred GC ending codons.

The codon adaptation index (CAI) is one measure that is used to estimate the extent of bias towards codons that are preferred in highly expressed genes. The CAI value ranges from 0 and 1.0 for a gene, where a higher value is likely to indicate stronger codon usage bias and a potential higher expression level. Higher CAI for a large set of genes may also indicate that selection for translation is active over that set of genes. Codons whose frequencies of usage were significantly higher in the genes with higher CAI, than that of the genes with lower CAI, are considered as the optimal codons. In this study, codon usage of retroviruses was compared (with chi-squared contingency test) between two groups of genes. One group of genes was constituted from $5 \%$ of the total number of genes, which had the maximum CAI values. The other group of genes was similarly constructed from the genes having minimum CAI. In all, 26 codons, UUU (Phe), UUA, UUG, CUA (Leu), AUA (Ile), GUA (Val), UAU (Tyr), CAU (His), CAA (Gln), AAU (Asn), AAA (Lys), GAU (Asp), GAA (Glu), UCU, UCA, AGU (Ser), CCU, CCA (Pro), ACU, ACA (Thr), GCU, GCA (Ala), UGU (Cys), AGA, AGG (Arg), and GGA (Gly), were identified as the optimal codons $(P<0.01)$ (Table 2). Almost all of these codons have an A or $U$ at the third position. It may be noted that the previously identified preferred codons are a subset of these optimal codons. Furthermore, it was found that significant correlations exist between CAI and ENc, AU and AU3 values $(r=0.23,-0.32$, -0.44 resp., $P<0.05)$ over the whole data set of retroviral genes. As expected if mutational bias is the main factor explaining codon usage bias in retroviruses, the frequency of preferred codons, as defined by most frequently used codons, is positively correlated with average AU composition.

3.3. Correspondence and Cluster Analysis. Correspondence analysis (CoA) on relative synonymous codon usage (RSCU) is a method for identifying major trends/factors (as orthogonal axes) responsible for the variation in codon usage among genes represented as 59- (number of sense codons) dimensional vectors. In the correspondence analysis on codon usage of retroviral genes, the two axes which accounted for the largest amount of variations, accounted for about $25 \%$ (major axis) and $10 \%$ of the variation of the whole data set. Each of the remaining axes accounted for less than $5 \%$ of the variation. The retroviral genes were widely distributed along the length of the first major axis. Genes belonging to differently biased viruses were distinctly separated on the first major axis. The AU rich retroviruses, for example, FIV, SFV3, VISNA, OLV, and HIV-1, were on the extreme right, while the GC rich viruses were on the other end (Figure 1(b)). It was interesting to note that Lentiviruses were closer to each other on the axis than to viruses from other retroviral genera. AU, AU3, and CAI correlated strongly with the first major axis $(r=0.89,0.9,-0.52$, resp., $P<0.05)$ (Figure $2(\mathrm{a}))$. The plot of the codons on first and second axes reveals that the A/Uending and G/C-ending synonymous codons are also clearly separated along the first major axis (Figure 2(b)).

Cluster analysis based on codon usage reveals the grouping within and across the organisms based on the similarities and differences in their codon usage. The organisms are grouped based on a distance measure which is proportional to the similarities of the codon usage between pairs of organisms. Cluster analysis on retroviral codon usage revealed that the retroviruses are grouped into two major clusters (Figure 3), the larger cluster being further divided into two subclusters. Alpharetroviruses with single genes constituted the minor cluster, while all the remaining viruses constituted the major cluster. It was observed that phylogenetically closely related retroviruses are relatively closer to each other in the clusters. Some retroviruses are relatively biased, grouped together in one subcluster. Retroviruses with higher AU and AU3 form one subcluster, while those with higher GC and GC3 form the other subcluster. It is seen that 
Euclidean distances

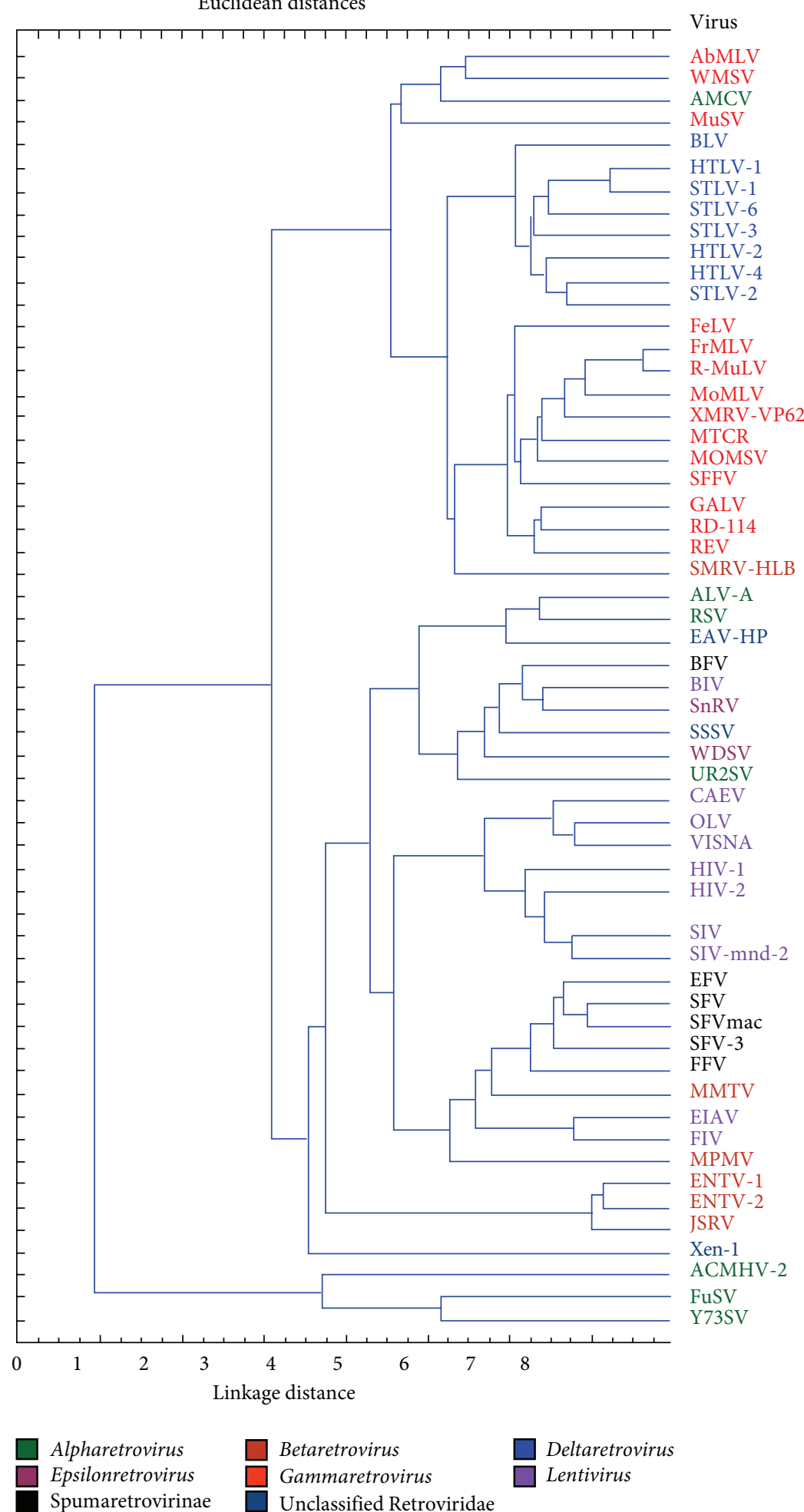

\begin{tabular}{|c|c|c|}
\hline ENc A & $\mathrm{AU}(\%)$ & AU 3 \\
\hline 55.03 & 46.73 & 46.50 \\
\hline 51.39 & 42.60 & 37.97 \\
\hline 52.79 & 39.60 & 32.00 \\
\hline 49.97 & 42.65 & 37.35 \\
\hline 52.05 & 44.98 & 47.35 \\
\hline 50.88 & 46.27 & 45.35 \\
\hline 51.09 & 46.73 & 45.80 \\
\hline 54.01 & 47.00 & 48.73 \\
\hline 55.39 & 44.92 & 44.52 \\
\hline 50.04 & 45.58 & 44.46 \\
\hline 50.86 & 42.85 & 40.68 \\
\hline 51.19 & 43.08 & 41.97 \\
\hline 53.84 & 50.30 & 53.60 \\
\hline 54.95 & 46.70 & 47.70 \\
\hline 55.42 & 46.87 & 47.60 \\
\hline 54.72 & 47.00 & 48.77 \\
\hline 52.79 & 46.43 & 46.80 \\
\hline 52.33 & 45.90 & 45.50 \\
\hline 56.40 & 47.60 & 46.08 \\
\hline 51.03 & 45.27 & 47.33 \\
\hline 55.83 & 47.57 & 48.83 \\
\hline 54.31 & 49.70 & 51.60 \\
\hline 57.09 & 46.90 & 46.50 \\
\hline 53.01 & 51.68 & 54.78 \\
\hline 57.09 & 44.80 & 45.23 \\
\hline 53.50 & 44.90 & 41.90 \\
\hline 59.38 & 47.60 & 47.80 \\
\hline 55.93 & 52.14 & 58.76 \\
\hline 53.22 & 53.26 & 57.90 \\
\hline 51.88 & 50.31 & 58.46 \\
\hline 54.69 & 51.70 & 57.90 \\
\hline 53.51 & 57.82 & 65.50 \\
\hline 55.68 & 53.40 & 56.65 \\
\hline 45.93 & 57.10 & 67.63 \\
\hline 44.59 & 57.72 & 65.73 \\
\hline 44.47 & 57.83 & 68.25 \\
\hline 45.05 & 55.49 & 64.78 \\
\hline 52.43 & 51.49 & 56.51 \\
\hline 48.80 & 54.73 & 60.62 \\
\hline 51.44 & 54.91 & 58.94 \\
\hline 45.53 & 58.00 & 71.16 \\
\hline 47.94 & 58.03 & 70.58 \\
\hline 46.02 & 58.52 & 73.60 \\
\hline 44.33 & 59.50 & 75.20 \\
\hline 48.58 & 60.53 & 70.40 \\
\hline 51.84 & 55.98 & 62.80 \\
\hline 47.05 & 59.25 & 67.43 \\
\hline 43.87 & 62.01 & 71.39 \\
\hline 50.10 & 57.58 & 68.13 \\
\hline 49.23 & 58.28 & 71.45 \\
\hline 49.86 & 57.63 & 70.20 \\
\hline 49.25 & 58.58 & 66.08 \\
\hline 59.60 & 53.35 & 58.00 \\
\hline 40.51 & 36.20 & 18.50 \\
\hline 42.61 & 37.90 & 22.20 \\
\hline 39.41 & 38.20 & 21.20 \\
\hline
\end{tabular}

FIGURE 3: Dendogram representing the extent of divergence in relative synonymous codon usage of 56 retroviruses, using unweighed pair group average clustering, and distances are in Euclidean distance. Different clades are in different colors. To the extreme right mean ENc, mean AU\% and AU3\% are added from Table 1. 
TABLE 2: Translational optimal codons.

\begin{tabular}{|c|c|c|c|c|c|}
\hline \multirow{2}{*}{ Amino acid } & \multirow{2}{*}{ Codon $^{\#}$} & \multicolumn{2}{|c|}{ High } & \multicolumn{2}{|c|}{ Low } \\
\hline & & RSCU & Number & RSCU & Number \\
\hline \multirow{2}{*}{ Phe } & UUU $^{*}$ & 1.64 & 184 & 0.7 & 61 \\
\hline & UUC & 0.36 & 40 & 1.3 & 113 \\
\hline \multirow{6}{*}{ Leu } & UUA $^{*}$ & 2.67 & 323 & 0.34 & 35 \\
\hline & UUG $^{*}$ & 1.21 & 146 & 0.5 & 51 \\
\hline & CUU & 0.47 & 57 & 0.63 & 65 \\
\hline & CUC & 0.29 & 35 & 1.83 & 189 \\
\hline & CUA $^{*}$ & 0.94 & 114 & 0.53 & 55 \\
\hline & CUG & 0.41 & 50 & 2.17 & 223 \\
\hline \multirow{3}{*}{ Ile } & AUU & 0.85 & 193 & 0.77 & 55 \\
\hline & AUC & 0.29 & 66 & 1.72 & 122 \\
\hline & AUA $^{*}$ & 1.86 & 425 & 0.51 & 36 \\
\hline \multirow{4}{*}{ Val } & GUU & 0.65 & 80 & 0.63 & 44 \\
\hline & GUC & 0.36 & 44 & 1.38 & 96 \\
\hline & GUA* $^{*}$ & 2.17 & 267 & 0.32 & 22 \\
\hline & GUG & 0.82 & 101 & 1.68 & 117 \\
\hline \multirow{2}{*}{ Tyr } & $\mathrm{UAU}^{*}$ & 1.74 & 270 & 0.44 & 34 \\
\hline & UAC & 0.26 & 41 & 1.56 & 121 \\
\hline \multirow{2}{*}{ His } & CAU $^{*}$ & 1.5 & 144 & 0.64 & 63 \\
\hline & CAC & 0.5 & 48 & 1.36 & 134 \\
\hline \multirow{2}{*}{ Gln } & $\mathrm{CAA}^{*}$ & 1.49 & 383 & 0.47 & 71 \\
\hline & CAG & 0.51 & 131 & 1.53 & 228 \\
\hline \multirow{2}{*}{ Asn } & AAU $^{*}$ & 1.67 & 317 & 0.54 & 38 \\
\hline & $\mathrm{AAC}$ & 0.33 & 62 & 1.46 & 104 \\
\hline \multirow{2}{*}{ Lys } & AAA $^{*}$ & 1.38 & 481 & 0.68 & 93 \\
\hline & AAG & 0.62 & 218 & 1.32 & 182 \\
\hline \multirow{2}{*}{ Asp } & GAU* $^{*}$ & 1.51 & 259 & 0.53 & 54 \\
\hline & GAC & 0.49 & 85 & 1.47 & 149 \\
\hline \multirow{2}{*}{ Glu } & GAA $^{*}$ & 1.5 & 474 & 0.61 & 99 \\
\hline & GAG & 0.5 & 159 & 1.39 & 228 \\
\hline \multirow{6}{*}{ Ser } & $\mathrm{UCU}^{*}$ & 1.18 & 77 & 0.76 & 49 \\
\hline & UCC & 0.56 & 37 & 2.1 & 136 \\
\hline & UCA $^{*}$ & 1.88 & 123 & 0.51 & 33 \\
\hline & UCG & 0.2 & 13 & 0.71 & 46 \\
\hline & $\mathrm{AGU}^{*}$ & 1.53 & 100 & 0.37 & 24 \\
\hline & AGC & 0.66 & 43 & 1.56 & 101 \\
\hline \multirow{4}{*}{ Pro } & $\mathrm{CCU}^{*}$ & 1.47 & 162 & 0.68 & 81 \\
\hline & CCC & 0.52 & 57 & 2.04 & 243 \\
\hline & $\mathrm{CCA}^{*}$ & 1.78 & 196 & 0.75 & 89 \\
\hline & CCG & 0.24 & 26 & 0.54 & 64 \\
\hline \multirow{4}{*}{ Thr } & $\mathrm{ACU}^{*}$ & 1.29 & 156 & 0.67 & 57 \\
\hline & ACC & 0.42 & 51 & 2.1 & 178 \\
\hline & $\mathrm{ACA}^{*}$ & 2.09 & 252 & 0.67 & 57 \\
\hline & ACG & 0.2 & 24 & 0.55 & 47 \\
\hline \multirow{4}{*}{ Ala } & $\mathrm{GCU}^{*}$ & 1.09 & 130 & 0.7 & 69 \\
\hline & GCC & 0.58 & 69 & 2.11 & 207 \\
\hline & $\mathrm{GCA}^{*}$ & 2.07 & 246 & 0.67 & 66 \\
\hline & GCG & 0.26 & 31 & 0.51 & 50 \\
\hline \multirow{2}{*}{ Cys } & $\mathrm{UGU}^{*}$ & 1.69 & 133 & 0.5 & 27 \\
\hline & UGC & 0.31 & 24 & 1.5 & 81 \\
\hline
\end{tabular}

TABLE 2: Continued.

\begin{tabular}{lccccc}
\hline \multirow{2}{*}{ Amino acid } & \multirow{2}{*}{ Codon $^{\#}$} & \multicolumn{2}{c}{ High } & \multicolumn{2}{c}{ Low } \\
& & RSCU & Number & RSCU & Number \\
\hline \multirow{4}{*}{ Arg } & CGU & 0.03 & 2 & 0.53 & 26 \\
& CGC & 0.09 & 6 & 1.64 & 81 \\
& CGA & 0.42 & 28 & 0.65 & 32 \\
& CGG & 0.12 & 8 & 2.03 & 100 \\
& AGA $^{*}$ & 3.71 & 250 & 0.45 & 22 \\
Gly & AGG $^{*}$ & 1.63 & 110 & 0.71 & 35 \\
& GGU & 0.49 & 73 & 0.42 & 37 \\
& GGC & 0.32 & 48 & 1.52 & 134 \\
& $G^{*}$ & 2.22 & 333 & 0.74 & 65 \\
& GGG & 0.97 & 145 & 1.33 & 117 \\
\hline
\end{tabular}

${ }^{\#} \mathrm{~W}, \mathrm{M}$, and stop codons are excluded. Those codons are significantly higher in highly expressed genes.

Lentiviruses are grouped with Betaretrovirus, Spumaretrovirinae, Epsilonretrovirus, some Alpharetrovirus, and unclassified retroviruses, which have higher AU and AU3. Delta and Gamma retroviruses exist in the other subcluster with GC and GC3 rich viruses. This observation was in accordance with the findings from $\mathrm{CoA}$.

\section{Discussion}

Retroviruses are an extremely important system for study, especially so because of its potential to adversely affect the quality of life and life-span of a large fraction of the world population especially in developing countries. These viruses are a potential threat to mankind, because of their complex biological mechanisms and evolution. This study aims to reveal the nature of some important genetic, genomic, and evolutionary features of these viruses which may be further utilized in better understanding of the retroviral system and has been designed to elucidate the general complexity and preferences of codon usage of all the retroviruses based on certain well-established parameters. Analysis of codon usage and base composition of retroviral genes documented here have revealed some useful facts. Furthermore, the results obtained through the various analyses were found to be consistent with each other, thus strongly validating the results obtained.

The large majority of the completely sequenced 56 retroviruses belonged to the Orthoretrovirinae subfamily. Within the Orthoretrovirinae, different genera contained almost equal number of viruses. Several features of retroviruses have been revealed through computation and analysis of different well-established parameters to understand their compositional and codon usage characteristics. They are RSCU, codon bias $(\mathrm{ENc})$, base content, preferred and optimal codons, major factors of $\mathrm{CoA}$, and grouping by cluster analysis of these viruses based on their codon usages.

It is found that retroviral genes do not possess significantly high codon bias. The genes are almost equally distributed between weak bias and moderate bias. This observation is very similar to the findings of Jenkins and Holmes, 
in 2003, where they had also observed moderate bias in 50 human RNA viruses [7]. However, the study by Jenkins and Holmes included only 4 human retroviruses, and in the present study a total of 56 completely sequenced retroviral sequences were analyzed, thus providing a more complete view of the total Retroviridae family that strongly supports the earlier observations by Jenkins and Holmes [7]. In addition, CoA was also performed in this present study that successfully discriminates between differently biased groups and added a new dimension in explaining the factors responsible for shaping the codon usage bias of the retroviruses. Large majority of the moderately biased genes belonged to the viruses from the Orthoretrovirinae subfamily. Among the total biased genes, most of the genes were from only FIV, SFV-3, VISNA, OLV, and HIV-1. There are large variations in the nucleotide composition of the retroviral genes. The AU content of the genes varied over a wide range of about $25 \%$ (range size), while the AU3 content varied over far larger range of 55\% (range size). This varying pattern of AU content in retroviruses is in good agreement with the earlier study by Jenkins and Holmes [7]. Four of the human retroviruses reported by Jenkins and Holmes and in this present study also followed similar base usage pattern [7]. Viruses, which exhibited higher codon usage bias (mentioned earlier), also possessed higher AU content, both at the third synonymous codon position and in overall gene composition. FIV had the highest AU and AU3 content over other retroviruses, while ACMHV-2 had the lowest. Though AU3 content increased with overall AU content, there was no specific nucleotide bias in the major fraction of first and second codon positions of retroviruses. AU content of the first and second codon positions combined is relatively higher in viruses which have relatively higher codon bias and higher AU \& AU3 content. There are some notable variations of base content of genes and codon bias even within retroviral genomes. Thus, heterogeneity of compositional bias exists both within and across retroviral genomes. Additionally, it is also clear from data that codon usage and base composition are virus-specific to a considerable extent. Majority of the genes were below the expected curve of codon bias when plotted against base composition of the third codon position (Figure 1(a)). This signifies that additional factors other than base composition might also have influenced retroviral codon usage.

Some retroviruses were significantly similar in their overall codon usage, while majority was not. Four preferred codons were identified, all of which were subset of the set of 26 optimal codons separately identified. It was observed that phylogenetically closer retroviruses possess relatively similar codon usage and almost the same sets of preferred and optimal codons having A or $\mathrm{U}$ in their synonymous positions. But Alpharetrovirus, Gammaretrovirus, and Deltaretrovirus were exceptions with relatively higher $\mathrm{G}$ or $\mathrm{C}$ in their 3rd codon positions. Compositions of the optimal codons were correlated with the average genetic base composition of these viruses. In correspondence analysis, the two dominant axes accounted for about one-third of the total variation of codon usage in retroviruses. It was seen that these axes successfully differentiated the genes based on codon bias, base content, and codon composition. Correlation between
CAI, as a measure of relative expression and first major axis, indicates that translational selection seemingly has a role in retroviruses. This observation implicates that once the viral genome enters into host translationary mechanism, then the biased genes (indicated by ENC and 1st major axis) having higher relative expression potential (higher CAI value) that matches the host can translate faster, thus achieving higher fitness for the virus [11]. These results and correlations between CAI, codon bias, and base composition indicate that genes with relatively higher codon bias are primarily composed of mostly optimal codons. Cluster analysis also validated that evolutionary-related retroviruses have similar codon usage, and those which are distant have distinctly different codon usage.

In the light of the general fact that selective constraints are greater in the first two positions of codons, whereas mutational bias is greater in the third position, all the observations indicate that codon bias in retroviruses in general is strongly dependent on base composition and mutational bias. This observation is also supported by earlier studies where it has been shown that main factor explaining codon usage in viruses is mutation bias $[7,12-14]$ and generally AT mutation bias in RNA viruses $[15,16]$. Selection for overall efficient expression for genes is probably an important factor affecting codon usage in these systems (as indicated by correlation between CAI and the first major axis). Groupings observed from cluster analysis and the conservation of preferred and optimal codons and similar base usage in phylogenetically close retroviruses indicate that codon usage and nucleotide composition might have evolved through a concerted process in these viral systems. There is a certain possibility that overall all AU richness of the retroviruses, being host dependent viruses, might have evolved due to differential cost and exploit the availability of relevant metabolites in the host cell [17]. This is also supported by the fact that small genome viruses, like retroviruses, are more AU prone than in contrast to large DNA viruses which are mostly GC rich [18]. Evidence of translational selection on codon usage bias in the viruses has been found on a subset of genes only, those for which selection efficiency or accuracy is possibly the most important for the survival of the viruses based on their improved expression $[19,20]$. This fact is also supported by earlier studies, where correlation between viral codon usage bias and host codon usage bias implicates better exploitation of the host translationary mechanism in cooperation with the host translational bias $[11,20]$.

There is a good possibility that compositional bias detected in retroviruses in this study is the result of a directional mutational pressure imposed by one of the two enzymes that copies the retroviral genome, that is, retrovirusspecific reverse-transcriptase (RT) enzyme, which converts the viral RNA into DNA. It is a distinct possibility that the absence of any strong codon bias in retroviruses might be due to the combined effect of missincorporations by the error-prone RT polymerase enzyme (mentioned above) and another class of enzyme, cytidine deaminases such as enzymes of APOBEC3 superfamily [21, 22] and lack of strong selection on codons in retroviral genes. There is indeed some experimental evidence that the HIV-1 RT enzyme is 
responsible for accumulation of A nucleotides in viral plusstrand genome. These enzymes are also supposed to be responsible for hypermutation of retroviruses, such as HIV1 and SIV [22, 23]. Relatively error-prone RT polymerase enzyme and enzymes like APOBEC3 cytidine deaminases preferentially incorporate $\mathrm{G}$ to $\mathrm{U}$ mismatches during minus strand cDNA synthesis [21,23] and eventually further induces $\mathrm{G}$ to A mutations in the viral RNA genome [24]. It is possible that other retroviruses also have evolved under the influence of similar enzymes, inducing hypermutation in those viruses. Relations observed in this study between distinct AU preference and codon usage bias in retroviruses may be due to this general propensity of $G$ to $U$ and further to A mutation. In fact, absence of relation between gene length and codon usage, implying the absence of strong selection for translational accuracy, specifically, in these viruses, might be due to the effect of missincorporations by the errorprone, RT polymerase itself [21] and cytidine deaminases [23]. Weak codon bias observed is possibly the result of these high mutation rates in retroviruses. This might be advantageous for maintaining high mutation rates in these viruses. Such rapid mutation, for example, in HIV-1, leads to an accumulation of diversity of its gene sequences. By diversifying, the viruses are probably able to escape host immune detection. But this phenomenon must be occurring at the expense of purifying effect of the general selection forces $[25,26]$. Furthermore, the weak codon bias in retroviruses might also be contributing towards decreasing host immune response during retroviral infection in the host by allowing the relatively lower expression of viral "env" to be suppressed in order to minimize antigenic profile of these viruses [27]. These critical processes may have shaped retroviral genes over time to become a very successful immunoinvading system. There are some lines of evidence that suggest that retroviruses, such as HIV-1, are subject to a positive selection pressure imposed by the immune system [28]. Additionally, previous studies indicate that retroviral gene expression is controlled by multiple complex regulatory mechanisms [29]. For example, HIV structural proteins are expressed from unspliced $9 \mathrm{~kb}$ (gag/pol) and partially spliced $4 \mathrm{~kb}$ (env) transcripts that are unstable and can efficiently be exported from the nucleus in absence of HIV regulatory protein Rev. The lack of nuclear stability and export in absence of Rev is partly due to the presence of defined inhibitory sequences (known as INS, IN, and CRS) within structural genes themselves. In this context, the low GC content of HIV RNA also contributes to nuclear instability, even in absence of defined inhibitory sequences [30]. All these facts may help to put in context the compositional patterns and codon usage bias in majority of retroviruses.

Observations from comparative analysis of codon usage bias reveal lack of strong translational selection in considerable number of retroviruses and this could be a problem of using retroviruses as expression vectors for gene therapy and immunization. Instead, use of the retroviruses with $\mathrm{AU}$ rich nucleotide composition is recommended, utilizing optimal set of codons. Information on optimal codons obtained from this study is expected to be useful for codon optimization especially for designing retroviral vectors with higher translational efficiency and production of simple and safe retroviral vectors for gene therapy and immunization.

\section{Conclusion}

Overall, the results point towards the fact that mutational bias is a dominant factor, relative to translational selection, in shaping codon usage of retroviruses. In these viruses, where codon usage bias is not strong, it is primarily determined by base composition, that is, AU (or GC) content of the genes, while selection for efficient expression for genes is probably another important factor affecting their codon usage. The intricate character of codon usage of these viral systems is probably maintained by incorporations of errors during molecular processing of the retroviral genomes, to help avoid strong immune response from the infected host but yet strike a balance with adequate execution of basic life cycle mechanisms of these viruses. In spite of inter- and intragenomic differences of base and codon usage, it is possible that the extant retroviruses, in general, have emerged through a complex but concerted process of evolution.

\section{Conflict of Interests}

The authors declare that they have no conflict of interests and that they did not receive financial support for this study.

\section{Acknowledgment}

The authors are thankful to Arpita Mukherjee (Scientist, Department of Electronics, Central Mechanical Engineering Research Institute, Durgapur-713209, India) for her technical help on statistics and critical reading of the paper.

\section{References}

[1] J. M. Coffin, "Retroviridae: the viruses and their replication," in Fields Virologyed, B. N. Fields, D. M. Knipe, and P. M. Howley, Eds., pp. 1767-1847, Lippincott-Raven, Philadelphia, Pa, USA, 3rd edition, 1996.

[2] R. A. Weiss, "How does HIV cause AIDS?" Science, vol. 260, no. 5112, pp. 1273-1279, 1993.

[3] R. Kurth and N. Bannert, Retroviruses: Molecular Biology. Genomics and Pathogenesis, Caister Academic Press, Berlin, Germany, 2010.

[4] M. Bulmer, "The selection-mutation-drift theory of synonymous codon usage," Genetics, vol. 129, no. 3, pp. 897-907, 1991.

[5] S. RoyChoudhury and D. Mukherjee, "A detailed comparative analysis on the overall codon usage pattern in herpesviruses," Virus Research, vol. 148, no. 1-2, pp. 31-43, 2010.

[6] L. Duret, "Evolution of synonymous codon usage in metazoans," Current Opinion in Genetics and Development, vol. 12, no. 6, pp. 640-649, 2002.

[7] G. M. Jenkins and E. C. Holmes, "The extent of codon usage bias in human RNA viruses and its evolutionary origin," Virus Research, vol. 92, no. 1, pp. 1-7, 2003.

[8] J. Peden, Analysis of Codon Usage, University of Nottingham, Department of Genetics, Nottingham, UK, 1997, http:// codonw.sourceforge.net. 
[9] J. O. McInerney, "GCUA: general codon usage analysis," Bioinformatics, vol. 14, no. 4, pp. 372-373, 1998.

[10] F. Wright, "The "effective number of codons" used in a gene," Gene, vol. 87, no. 1, pp. 23-29, 1990.

[11] A. Carbone, "Codon bias is a major factor explaining phage evolution in translationally biased hosts," Journal of Molecular Evolution, vol. 66, no. 3, pp. 210-223, 2008.

[12] I. S. Belalov and A. N. Lukashev, "Causes and implications of codon usage bias in RNA viruses," PLoS ONE, vol. 8, no. 2, Article ID e56642, 2013.

[13] Y. Zhang, Y. Liu, W. Liu et al., "Analysis of synonymous codon usage in hepatitis A virus," Virology Journal, vol. 8, article 174, 2011.

[14] S. Roychoudhury, A. Pan, and D. Mukherjee, "Genus specific evolution of codon usage and nucleotide compositional traits of poxviruses," Virus Genes, vol. 42, no. 2, pp. 189-199, 2011.

[15] Q. Zhong, W. Xu, Y. Wu, and H. Xu, "Patterns of synonymous codon usage on human metapneumovirus and its influencing factors," Journal of Biomedicine and Biotechnology, vol. 2012, Article ID 460837, 7 pages, 2012.

[16] N. Goni, A. Iriarte, V. Comas et al., "Pandemic influenza A virus codon usage revisited: biases, adaptation and implications for vaccine strain development," Virology Journal, vol. 9, article 263, 2012.

[17] E. P. C. Rocha and A. Danchin, "Base composition bias might result from competition for metabolic resources," Trends in Genetics, vol. 18, no. 6, pp. 291-294, 2002.

[18] L. A. Shackelton, C. R. Parrish, and E. C. Holmes, "Evolutionary basis of codon usage and nucleotide composition bias in vertebrate DNA viruses," Journal of Molecular Evolution, vol. 62, no. 5, pp. 551-563, 2006.

[19] E. Cowe and P. M. Sharp, "Molecular evolution of bacteriophages: discrete patterns of codon usage in T4 genes are related to the time of gene expression," Journal of Molecular Evolution, vol. 33, no. 1, pp. 13-22, 1991.

[20] S. Michely, E. Toulza, L. Subirana et al., "Evolution of codon usage in the smallest photosynthetic eukaryotes and their giant viruses," Genome Biology and Evolution, vol. 5, no. 5, pp. 848859, 2013.

[21] J. Vartanian, A. Meyerhans, M. Sala, and S. Wain-Hobson, "G $\rightarrow$ A hypermutation of the human immunodeficiency virus type 1 genome: evidence for dCTP pool imbalance during reverse transcription," Proceedings of the National Academy of Sciences of the United States of America, vol. 91, no. 8, pp. 3092-3096, 1994.

[22] A. Takaori-Kondo, "APOBEC family proteins: novel antiviral innate immunity," International Journal of Hematology, vol. 83, no. 3, pp. 213-216, 2006.

[23] K. Sato, T. Izumi, N. Misawa et al., "Remarkable lethal Gto-A mutations in vif-proficient HIV-1 provirus by individual APOBEC3 proteins in humanized mice," Journal of Virology, vol. 84, no. 18, pp. 9546-9556, 2010.

[24] B. Brekhout, A. T. Das, and N. Beerens, "HIV-1 RNA editing, hypermutation and error-prone reverse transcription," Science, vol. 292, no. 5514, article 7, 2001.

[25] L. M. Mansky, "Retrovirus mutation rates and their role in genetic variation," Journal of General Virology, vol. 79, no. 6, pp. 1337-1345, 1998.

[26] R. Shankarappa, J. B. Margolick, S. J. Gange et al., "Consistent viral evolutionary changes associated with the progression of human immunodeficiency virus type 1 infection," Journal of Virology, vol. 73, no. 12, pp. 10489-10502, 1999.
[27] C. Williamson, L. Morris, M. F. Maughan et al., "Characterization and selection of HIV-1 subtype C isolates for use in vaccine development," AIDS Research and Human Retroviruses, vol. 19, no. 2, pp. 133-144, 2003.

[28] H. A. Ross and A. G. Rodrigo, "Immune-mediated positive selection drives human immunodeficiency virus type 1 molecular variation and predicts disease duration," Journal of Virology, vol. 76, no. 22, pp. 11715-11720, 2002.

[29] H. Tang, K. L. Kuhen, and F. Wong-Staal, "Lentivirus replication and regulation," Annual Review of Genetics, vol. 33, pp. 133-170, 1999.

[30] K. Nguyen, M. Llano, H. Akari et al., "Codon optimization of the HIV-1 vpu and vif genes stabilizes their mRNA and allows for highly efficient Rev-independent expression," Virology, vol. 319 , no. 2, pp. 163-175, 2004. 


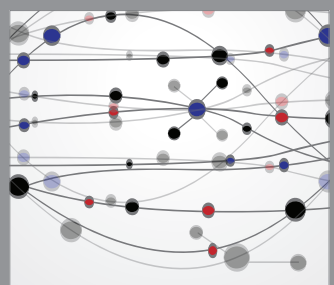

The Scientific World Journal
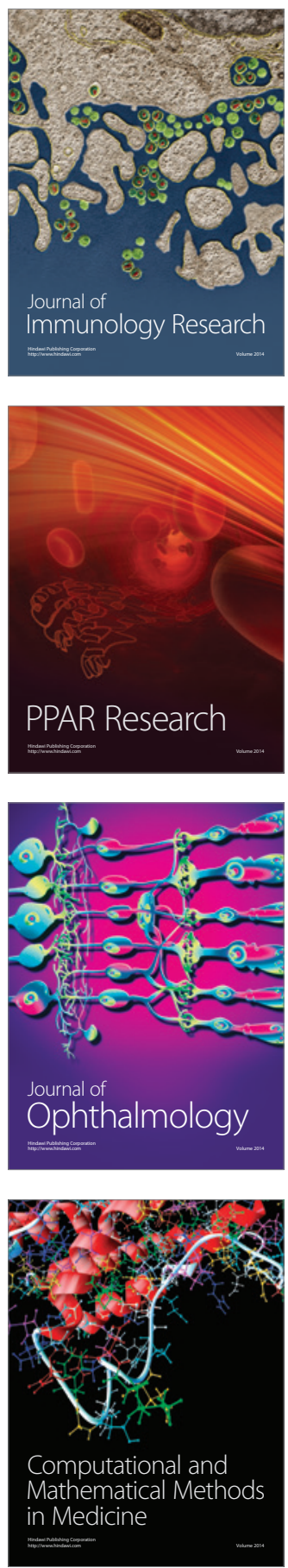

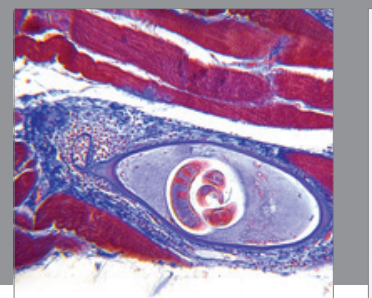

Gastroenterology

Research and Practice
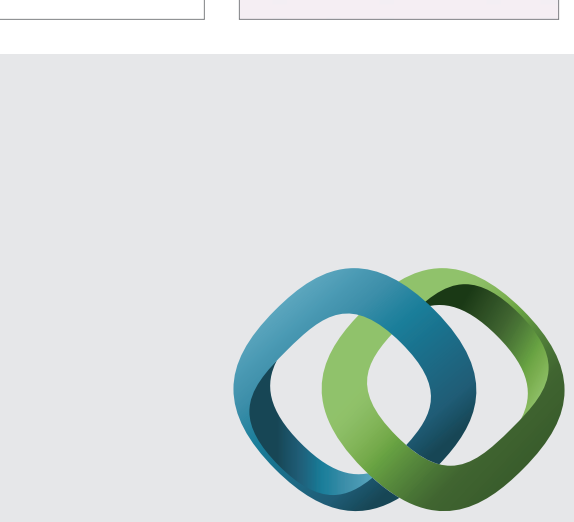

\section{Hindawi}

Submit your manuscripts at

http://www.hindawi.com
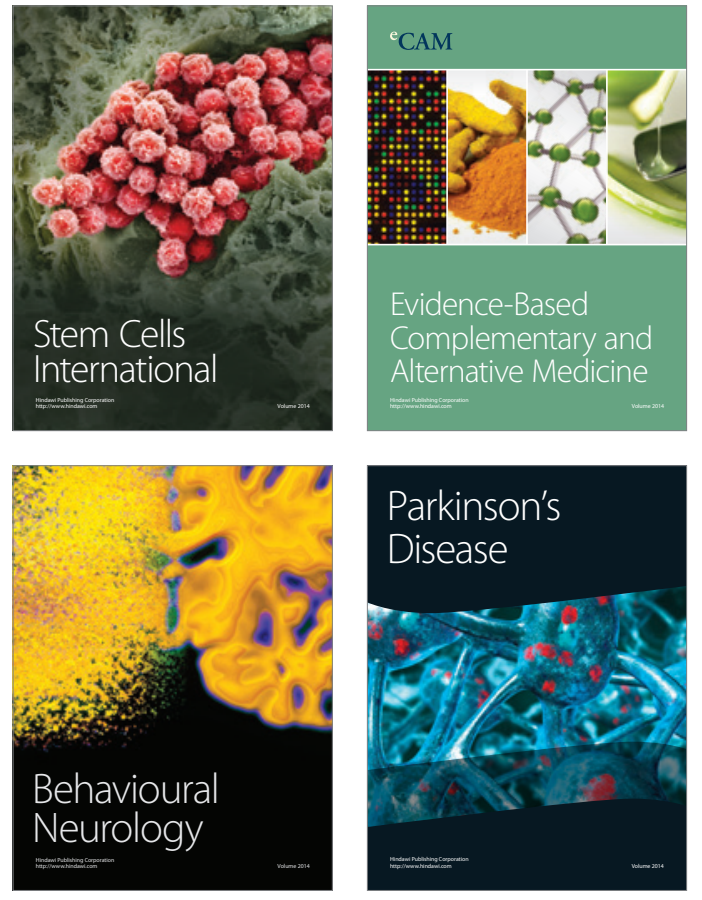
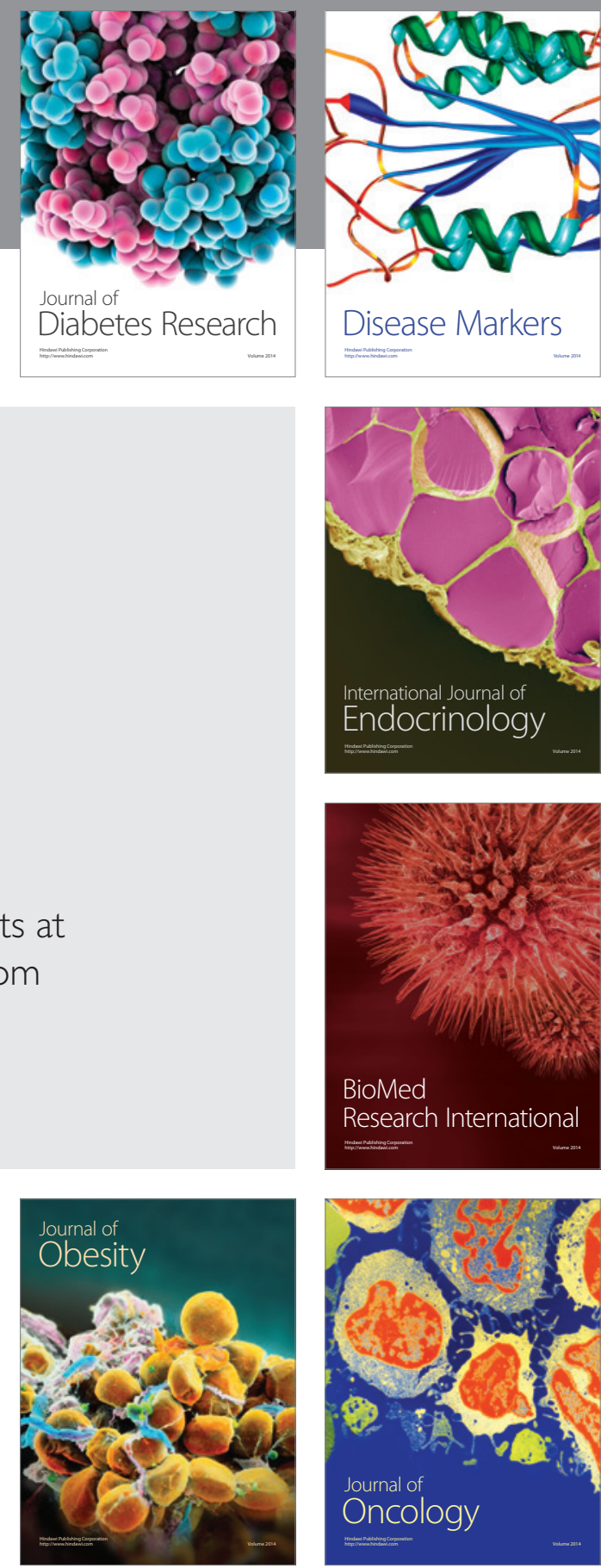

Disease Markers
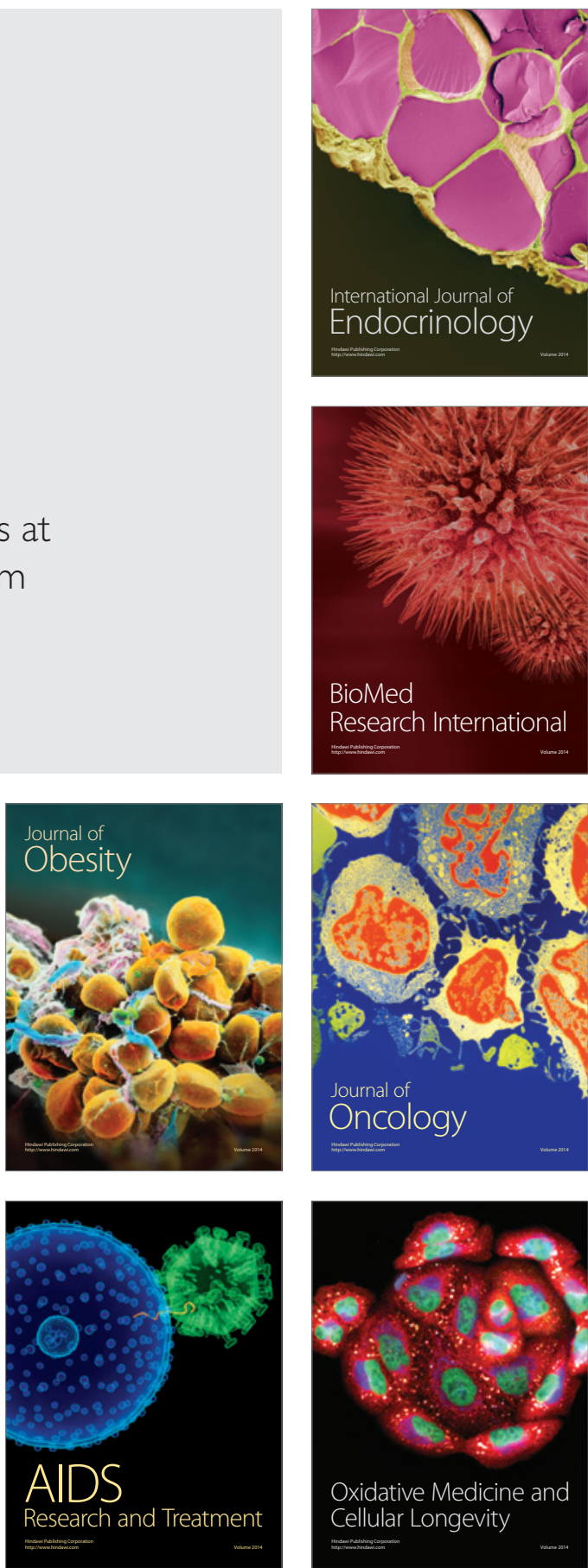